\title{
Parabolic Implosion
}

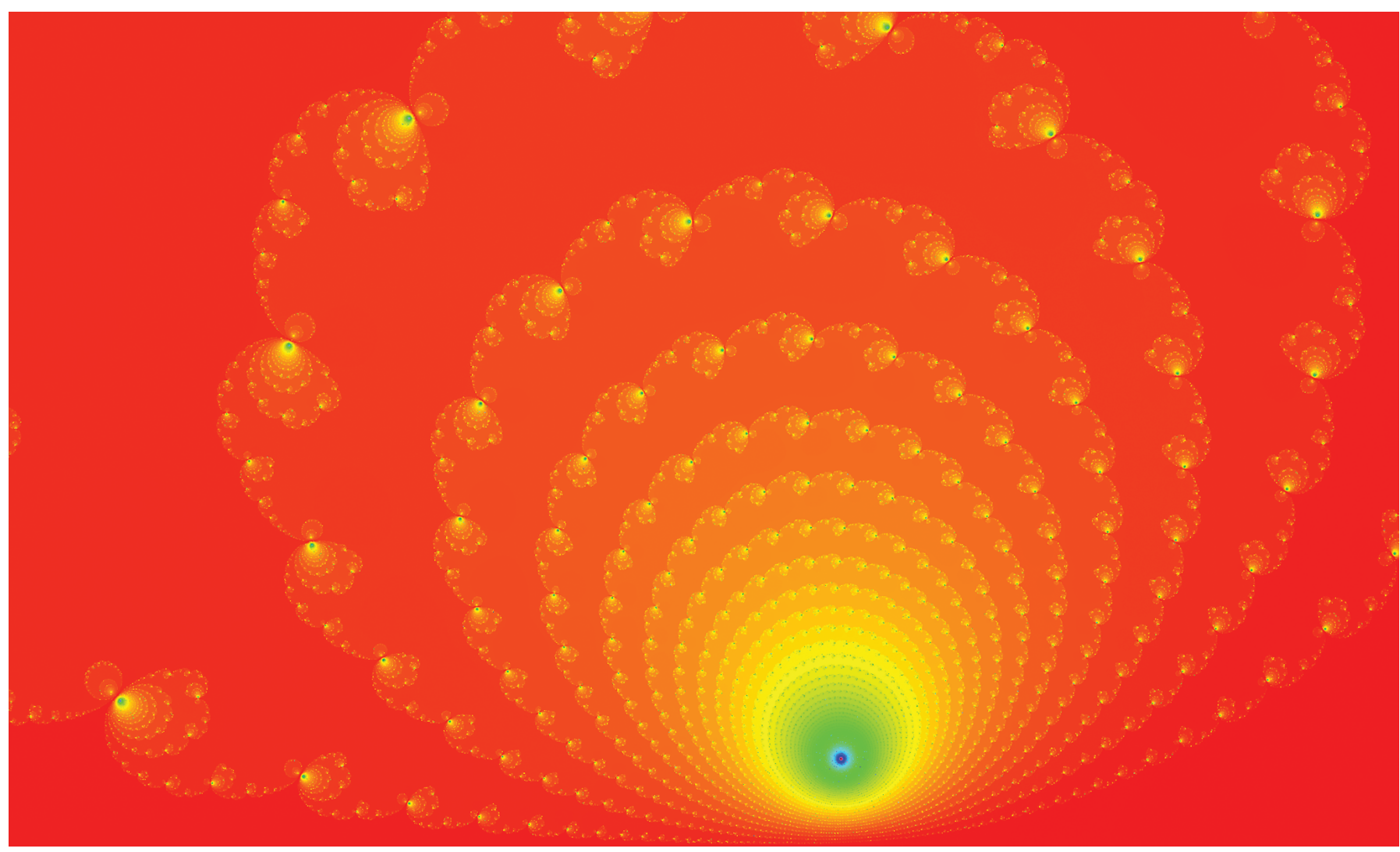

\section{Han Peters and Liz Vivas}

\section{Introduction}

In discrete dynamical systems one considers orbits of selfmaps $f: X \rightarrow X$. More generally one can consider a family of selfmaps $f_{\lambda}: X \rightarrow X$, depending on one or more parameters $\lambda \in \Lambda$. A central topic in dynamical systems is the dependence of dynamical behavior on the parameter: do small changes in $\lambda$ lead to wildly different dynamical behavior? Or does the opposite hold: are all nearby maps in some sense qualitatively similar?

The strongest form of qualitative similarity that one can hope for is for nearby maps to be conjugate. The selfmap $f_{\lambda_{0}}$ is structurally stable if for all nearby $\lambda \sim \lambda_{0}$ the map $f_{\lambda}$ is

Han Peters is a professor at the University of Amsterdam. His email address is h.peters@uva.n1.

Liz Vivas is an associate professor at the Ohio State University. Her email address is vivas@math.osu.edu.

Communicated by Notices Associate Editor Stephan Ramon Garcia.

For permission to reprint this article, please contact:

reprint-permission@ams.org.

DOI: https://doi.org/10.1090/noti2132 conjugate to $f_{\lambda_{0}}$; i.e., $f_{\lambda}=\varphi \circ f_{\lambda_{0}} \circ \varphi^{-1}$ for some invertible selfmap $\varphi: X \rightarrow X$. When this is the case we may also say that the parameter value $\lambda_{0}$ is structurally stable.

Structural stability is typically known to hold for systems that are hyperbolic, in the sense of being uniformly expanding/contracting. If there is interference between expanding and contracting behavior, or if there are invariant sets on which the dynamical behavior is neutral, i.e., neither uniformly expanding nor contracting, then structural stability may fail.

In one-dimensional complex dynamics structural stability has been extensively investigated. An analytic family of holomorphic maps $f_{\lambda}$ is structurally stable near a parameter $\lambda_{0}$ if and only if the Julia set of $f_{\lambda}$ varies continuously at $\lambda_{0}$, a result due to Mañé, Sad, and Sullivan [15]. Of particular importance is the behavior near a parameter $\lambda_{0}$ for which $f_{\lambda_{0}}$ has a parabolic cycle: a neutral periodic cycle whose multiplier is a root of unity. These parameter values are far from structurally stable. Paradoxically, the limit dynamical behavior of sequences $f_{\lambda}$ for which $\lambda \rightarrow \lambda_{0}$ is quite 
well understood, and lies on the basis of many of the most prominent results in the field.

In this note we will describe the phenomenon known as "parabolic implosion" and sketch a proof of its occurrence. We will describe the role of parabolic implosion in the proofs of several classical results in the field. We finish by discussing the status of parabolic implosion in complex dynamical systems in higher dimensions, and its relevance for recent breakthroughs in the area.

\section{Varying Julia Sets}

For simplicity we will now only consider rational functions $f: \hat{\mathbb{C}} \rightarrow \hat{\mathbb{C}}$, where $\hat{\mathbb{C}}$ stands for the Riemann sphere. The Fatou set of $f$, which we denote by $\mathcal{F}(f)$, consists of all $z \in \hat{\mathbb{C}}$ for which the family $\left\{f^{\circ n}\right\}$ is a normal family on an open neighborhood of $z$, where we use $f^{\circ n}$ to denote the $n$th iterate of $f$. Recall that a family of maps is said to be normal if it is equicontinuous or, equivalently, by the Arzelà-Ascoli theorem, if it is precompact; i.e., every sequence has a uniformly convergent subsequence. The Julia set $J(f)$ is the complement of the Fatou set.

Many interesting dynamical phenomena (though certainly not all) already occur for the seemingly simple family of quadratic polynomials. After conjugation with an affine map, a quadratic polynomial can be reduced to the form $p_{c}(z)=z^{2}+c$. We describe three possible behaviors of quadratic polynomials via the relative simple examples $c=0, c=-1$, and $c=1 / 4$.

When $c=0$ the Julia set of $p_{0}(z)=z^{2}$ is the unit circle. The Fatou set consists of two connected components: the basins of the attracting fixed points at 0 and $\infty$. A fixed point is a solution of the equation $z=p_{c}(z)$. We say a fixed point is attracting if $\left|p_{c}^{\prime}(z)\right|<1$, and repelling when $\left|p_{c}^{\prime}(z)\right|>1$. When $\left|p_{c}^{\prime}(z)\right|=1$ we say that the fixed point $z$ is neutral. Since fixed points are solutions of the equation $p_{c}(z)-z=0$, the implicit function theorem implies that fixed points $z$ with $p_{c}^{\prime}(z) \neq 1$ depend locally analytically on the parameter $c$.

For $0<r<1$ observe that the disk $B(0, r):=\{|z-0|<r\}$ is mapped strictly inside itself by $p_{0}$, and similarly for $B(\infty, r):=\left\{|z|>\frac{1}{r}\right\}$, the "disk" centered at $\infty$. This behavior is stable under perturbations: for sufficiently nearby $f \sim p_{0}$ (using the supremum on compacts norm for example) both $B(0, r)$ and $B(\infty, r)$ are still mapped strictly into themselves; hence all orbits in each of these disks converge to an attracting fixed point. It follows that the Julia set $J_{f}$ varies continuously (in the Hausdorff metric, discussed below) near $p_{0}$. Note that structural stability holds regardless of whether we consider perturbations $f$ in the class of quadratic polynomials or in the class of rational functions.

When $c=-1$ the Fatou set again consists of two attracting basins: the basin of the attracting fixed point at $\infty$, and the basin of the attracting 2 -cycle $\{0,-1\}$. The latter consists

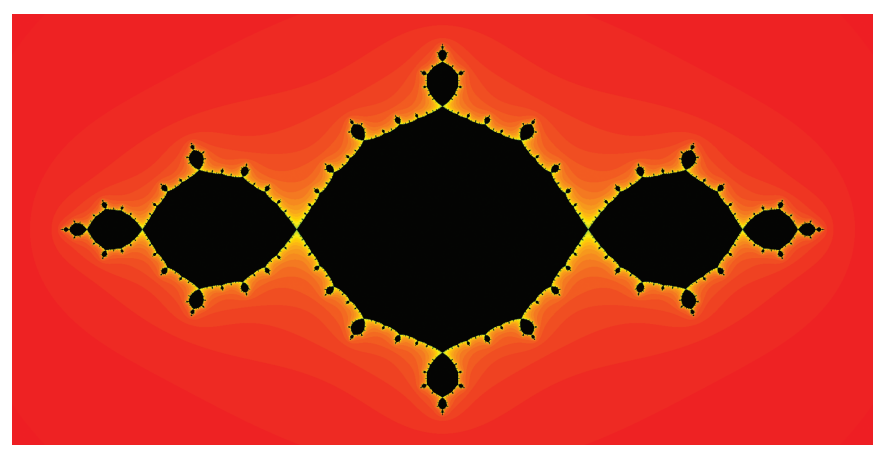

Figure 1. $c=-1$.

of countably many connected components (see Figure 1), but regardless the above argument still holds, and the map $p_{-1}$ is structurally stable.

When $c=\frac{1}{4}$ the Fatou set once more consists of two connected components: the basin of the attracting fixed point at $\infty$, and the basin of the parabolic fixed point $z=$ $1 / 2$; see Figure 2. A fixed point $z=f(z)$ is called parabolic if $f^{\prime}(z)$ is a root of unity. By considering a suitable iterate one can always reduce to the case $f^{\prime}(z)=1$, a situation already satisfied for the first iterate of $p_{1 / 4}$.

Observe that we can write

$$
p_{1 / 4}(z)=z+\left(z-\frac{1}{2}\right)^{2} ;
$$

hence $\frac{1}{2}$ is a double solution of the equation $f(z)-z=0$. This double solution splits up into two separate solutions for $c \neq 1 / 4$, which do not vary analytically at $c=1 / 4$. It turns out that there exist arbitrarily small perturbations $c \sim$ $1 / 4$ for which the Julia set of $p_{c}$ is not a small perturbation of $J\left(p_{1 / 4}\right)$.

Figure 3 illustrates the dynamics of a conveniently chosen perturbation $p_{c}$, with $c \sim 0.25$ (the precise value of $c$ is $0.250039+0.000000315817 i$; see Section 5 for details). The constant $c$ lies outside of the Mandelbrot set, which implies that the Julia set is totally disconnected and the Fatou set is a single connected component: the basin of infinity (see section 6 for precise definitions). Observe that the outline in the figure is almost identical to that of Figure 2, but the large black component, the "parabolic basin," has disappeared. In fact, the small black spots that are still visible are faulty artifacts of the fact that the computer uses only a finite number of iterates. The computer determines for each chosen initial value whether the first 10,000 points of its orbit stay in a disk of given radius. If it does, the initial value is drawn in black; if not, the color of the initial value depends on the number of iterates needed to leave the disk.

Near each of the black spots one can clearly see yellow spirals in what used to be the parabolic basin. The yellow is contained in the Fatou set, but indicates the presence of nearby points in the Julia set, referred to as embellishments. 


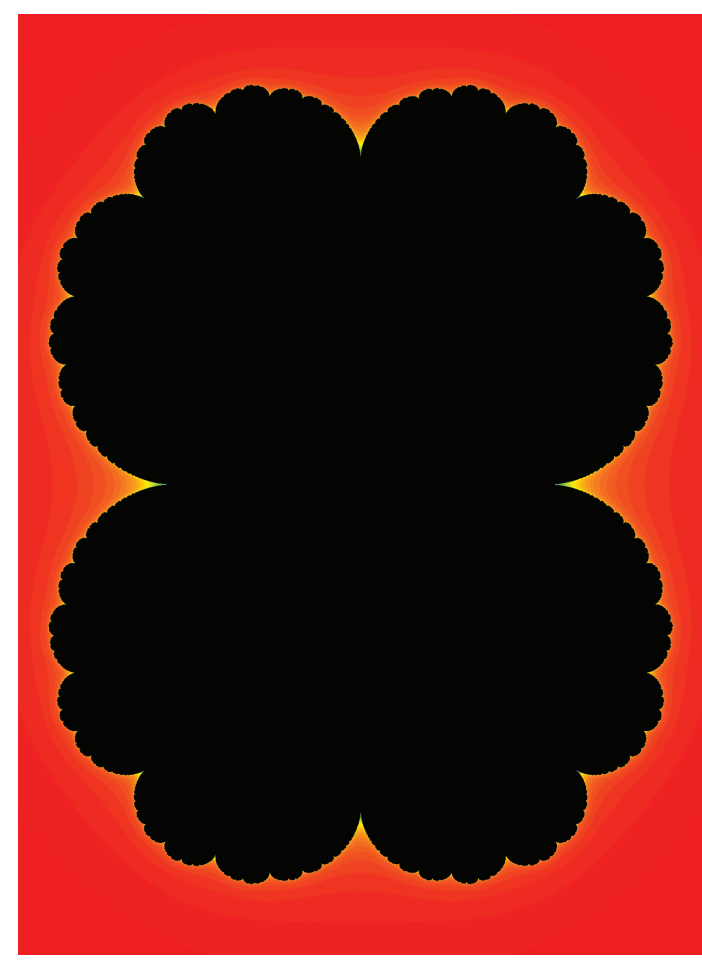

Figure 2. $c=\frac{1}{4}$.

A consequence of the Lavaurs Theorem, discussed below, is that the embellishments do not vanish for a certain sequence of $c^{\prime}$ s approaching $1 / 4$, which implies that the Julia set does not vary continuously near $1 / 4$ and the map $p_{1 / 4}$ is not structurally stable.

It was proved by Mañé, Sad, and Sullivan [15] that the Julia set of $p_{c}$ varies continuously near $c_{0} \in \mathbb{C}$ if and only if $p_{c_{0}}$ contains no neutral periodic points, i.e., periodic points $z=p_{c_{0}}^{k}(z)$ for which $\left|\left(p_{c_{0}}^{k}\right)^{\prime}(z)\right|=1$. For more general families of rational functions, one should consider "nonpersistent" neutral periodic points.

Instead of giving a precise definition of a persistent neutral cycle, we give a trivial example demonstrating that a neutral cycle does not need to be an obstruction to structural stability. Note that each map in the family $g_{d}(z)=$ $z+(z-d)^{2}$ has a parabolic fixed point at the point $d$, which of course does depend analytically on the parameter. The maps $g_{d}$ are all conjugate to $g_{0}$ via the translations $z \mapsto z+d$, and the Julia set therefore varies continuously. Persistent neutral cycles cannot occur for the quadratic family $\left\{p_{c}\right\}$.

\section{Semicontinuity}

Let us recall the topology on the set of compact subsets of a metric space $(X, d)$. Given two compact subsets $A, B \in X$ we define

$$
d(A, B):=\sup _{a \in A} \inf _{b \in B} d(a, b) .
$$

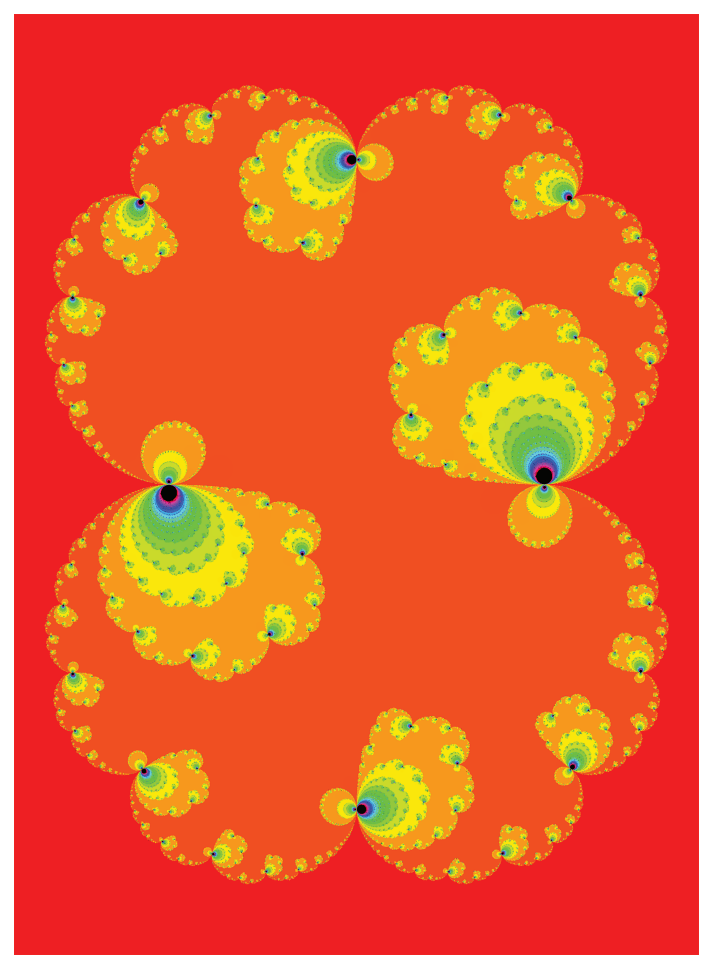

Figure 3. $c \sim 0.25$.

We note that $d(\cdot, \cdot)$ is not symmetric, and therefore not a metric. To fix this we define

$$
d_{H}(A, B):=\max \{d(A, B), d(B, A)\},
$$

which is symmetric and known as the Hausdorff metric. When we talk about the possible (dis)continuity of the Julia set, it is with respect to the topology induced by $d_{H}$. Denote by $\operatorname{Comp}(X)$ the set of compact subsets of $X$. A map $f: \Lambda \rightarrow \operatorname{Comp}(X)$ is upper semicontinuous at $\lambda_{0}$ if $d\left(f(\lambda), f\left(\lambda_{0}\right)\right) \rightarrow 0$ when $\lambda \rightarrow \lambda_{0}$, and it is lower semicontinuous if $d\left(f\left(\lambda_{0}\right), f(\lambda)\right) \rightarrow 0$ when $\lambda \rightarrow \lambda_{0}$.

As illustrated in Figures 2 and 3, the Julia set does not necessarily depend continuously on the parameter. However, the Julia set always depends lower semicontinuously. To see this, we recall that repelling periodic points are dense in the Julia set, a fact proved independently (and with different proofs) by Julia and Fatou. Given a rational function $f_{0}$, we can therefore find a large but finite set of repelling periodic points that are arbitrarily close to each point in $J\left(f_{0}\right)$. Each of these finitely many periodic points varies continuously by the implicit function theorem, hence for $f_{\lambda}$ sufficiently close to $f_{0}$ there are still repelling periodic points close to any point in $J\left(f_{0}\right)$. Since these perturbed repelling periodic points must lie in $J\left(f_{\lambda}\right)$, it follows that $d\left(J\left(f_{0}\right), J\left(f_{\lambda}\right)\right)$ is arbitrarily small.

We note that this argument does not imply that $d\left(J\left(f_{\lambda}\right), J\left(f_{0}\right)\right)$ is small, which is not necessarily the case. In fact, consider again the polynomial $p_{1 / 4}(z)=z^{2}+1 / 4$, and let $w_{0}$ be any point in the interior of the black set 
depicted in Figure 2. This black set, informally called the "cauliflower," is the filled Julia set $K\left(p_{1 / 4}\right)$, and its boundary is the Julia set $J\left(p_{1 / 4}\right)$. It turns out that there are arbitrarily small perturbations $c$ of $1 / 4$ for which $w_{0} \in J\left(p_{c}\right)$. The set of all accumulation points of sets $J\left(p_{c}\right)$, as $c \rightarrow 1 / 4$, is therefore equal to the black set $K\left(p_{1 / 4}\right)$, the cauliflower region enclosed by $J\left(p_{1 / 4}\right)$. Informally we say that the Julia set of the parabolic function $p_{1 / 4}$ implodes under small perturbations, a phenomenon referred to as parabolic implosion. Note that it is not the Julia set that implodes; the Julia set becomes larger. It is the connected component of the Fatou set (the cauliflower) that implodes.

In order to obtain a better understanding of the implosion phenomenon we will introduce two additional concepts: Fatou coordinates and the Lavaurs Theorem.

\section{Fatou Coordinates}

Key ingredients in our understanding of the dynamics near a parabolic fixed point, and thus necessary for understanding parabolic implosion, are the so-called Fatou coordinates, sometimes referred to as Leau-Fatou coordinates.

While the description holds for maps that are only locally defined, for simplicity we will consider a polynomial $f$ of degree at least 2 with a parabolic fixed point. Without loss of generality we may assume that this fixed point is the origin; hence we can write the Taylor series expansion

$$
f(z)=z+a_{2} z^{2}+a_{3} z^{3}+\cdots .
$$

Since we assumed that the degree of $f$ is at least 2 , there exists a minimal $k \geq 1$ for which $a_{k+1} \neq 0$. The number $k$ is referred to as the order of the parabolic fixed point. Let us first consider the case $k=1$. By conjugating with a linear map we may then assume that $f$ is of the form

$$
f(z)=z+z^{2}+a_{3} z^{3}+\cdots .
$$

Observe that for $r>0$ small enough the open disk $B(-r, r)$, whose boundary contains the origin, is mapped into itself by $f$. All the orbits in this disk converge to the origin, tangentially to the negative real axis. The disk $B(-r, r)$ is called a parabolic petal. For a set $U \subset \mathbb{C}$, we write $f^{-n}(U)=\left\{z \in \mathbb{C} ; f^{n}(z) \in U\right\}$. The set of all points whose orbits land in the petal,

$$
\bigcup_{n \in \mathbb{N}} f^{-n}(B(-r, r))
$$

is called the parabolic basin, denoted by $\mathcal{B}_{f}$. Note that the petal is not unique, but the basin is; it does not depend on $r$.

Conjugating $f$ on the petal with the change of coordinates $u=-\frac{1}{z}$, we obtain a map of the form

$$
u \mapsto u+1+r(u),
$$

defined on the half-plane $\operatorname{Re}(u)>\frac{1}{2 r}$, where $|r(u)| \rightarrow 0$ as $u \rightarrow \infty$. Leau and Fatou proved that we can further change coordinates to obtain the map

$$
u \mapsto u+1 \text {. }
$$

By pulling back the change of coordinates by the dynamics, we can extend the change of coordinates holomorphically to the entire parabolic basin, obtaining a $\operatorname{map} \varphi_{f}: \mathcal{B}_{f} \rightarrow$ $\mathbb{C}$ satisfying the Abel functional equation:

$$
\left(\varphi_{f} \circ f\right)(z)=\varphi_{f}(z)+1 .
$$

We refer to $\varphi_{f}$ as the incoming Fatou coordinate. By considering the inverse map, which is only locally defined, we can define $\varphi_{f^{-1}}$, a transformation that maps an outgoing petal $B(r, r)$ to roughly a left half-plane, and conjugates $f^{-1}$ to a translation by -1 . The transformation $\varphi_{f^{-1}}$ cannot be globally extended, since $f^{-1}$ is not globally defined. However, the inverse of $\varphi_{f^{-1}}$, which we will denote by $\psi_{f}: \mathbb{C} \rightarrow \mathbb{C}$, can be extended by use, of the forward dynamics of $f$ to a globally defined map, the outgoing Fatou coordinate. The map $\psi_{f}$ satisfies the following Abel equation:

$$
\psi_{f}(Z+1)=f \circ \psi_{f}(Z) .
$$

(Here we use the variable $Z$ to indicate we are working now with the inverse map of $\varphi$.)

For a polynomial with a parabolic fixed point, both $\varphi_{f}$ and $\psi_{f}$ are surjective.

For parabolic points of higher order $k \geq 2$ we can similarly define $k$ parabolic petals, each with its corresponding parabolic basin and incoming Fatou component. By considering the inverse map one can also define $k$ outgoing Fatou coordinates. Consider Figure 4 for an illustration of the distinct parabolic basins for a parabolic fixed point of order 3.

For fixed points of a polynomial $p$ with derivative a root of unity we can instead consider a suitable iterate of $p$ in order to reduce to the case of derivative 1 . The same trick can be used for parabolic periodic cycles.

We conclude this section by introducing the Lavaurs maps, which will play a vital role in the next section. We restrict our attention again to a polynomial $f$ with a parabolic fixed point of order $k=1$, and denote the corresponding incoming and outgoing Fatou coordinates by $\varphi_{f}$ and $\psi_{f}$. Let $\alpha \in \mathbb{C}$ and denote by $\tau_{\alpha}$ the translation $z \mapsto z+\alpha$. The Lavaurs map of phase $\alpha$ is defined by

$$
\mathcal{L}_{\alpha, f}:=\psi_{f} \circ \tau_{\alpha} \circ \varphi_{f} .
$$

We remark that $\mathcal{L}_{\alpha, f}$ maps the parabolic basin $\mathcal{B}_{f}$ surjectively to the entire complex plane $\mathbb{C}$. The discussion in this section holds just as well for rational functions, except that in that case the image $\mathcal{L}_{\alpha, f}\left(\mathcal{B}_{f}\right)$ avoids at most a single point in the Riemann sphere $\widehat{\mathbb{C}}=\mathbb{C} \cup\{\infty\}$.

\section{Lavaurs Theorem}

Let $f$ be a rational function with a parabolic fixed point at the origin, given locally by a power series expansion 


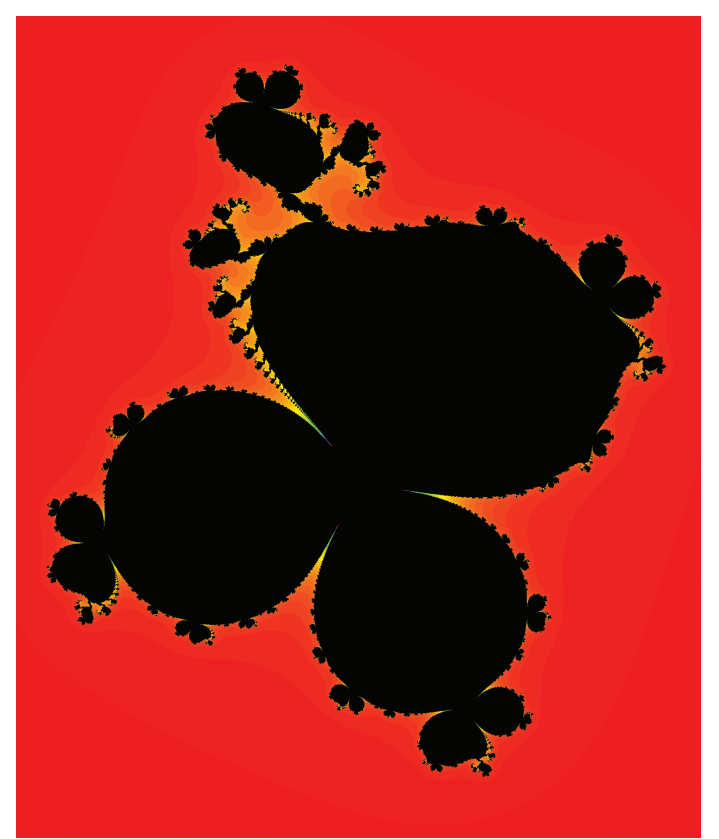

Figure 4. Parabolic fixed point of order 3.

$f(z)=z+z^{2}+O\left(z^{3}\right)$. We are interested in an analytic one-parameter family of rational functions containing $f$, for which the parabolic fixed point is nonpersistent. We consider the specific one-parameter family given by

$$
f_{\epsilon}(z)=f(z)+\epsilon^{2}
$$

for $\epsilon \in \mathbb{C}$.

Theorem 1 (Lavaurs). Let $\alpha \in \mathbb{C}$, and consider two sequences $N_{j} \in \mathbb{N}$ and $\epsilon_{j} \in \mathbb{C}$ satisfying

$$
N_{j}-\frac{\pi}{\epsilon_{j}} \rightarrow \alpha
$$

as $j \rightarrow \infty$. Then

$$
\left(f_{\epsilon_{j}}\right)^{\circ N_{j}} \rightarrow \mathcal{L}_{\alpha, f}
$$

where the convergence is locally uniformly on $\mathcal{B}_{f}$.

For a complete proof of the theorem above, the reader can consult the paper by Shishikura [17].

We can now explain the choice of the map $f$ used to generate Figure 3. Given any $\alpha \in \mathbb{C}$ we can for any $N \in \mathbb{N}$ choose $\epsilon$ such that the equation

$$
N-\frac{\pi}{\epsilon}=\alpha
$$

is satisfied, i.e., $\epsilon=\pi /(N-\alpha)$. The Julia set of $f_{\epsilon}$ equals the Julia set of $f_{\epsilon}^{\circ N}$. Hence for large $N$ we iterate in effect a very small perturbation of $\mathcal{L}_{\alpha}$ on $\mathcal{B}_{f}$, with the understanding that any orbit landing well outside of $\mathcal{B}_{f}$ will converge to infinity under iteration of $f_{\epsilon}$. For generating Figure 3 we used $f=p_{1 / 4}, \alpha=2 i$, and $N=500$, which gives

$$
f_{\epsilon}(z)=z^{2}+0.250039+0.000000315817 i \text {. }
$$

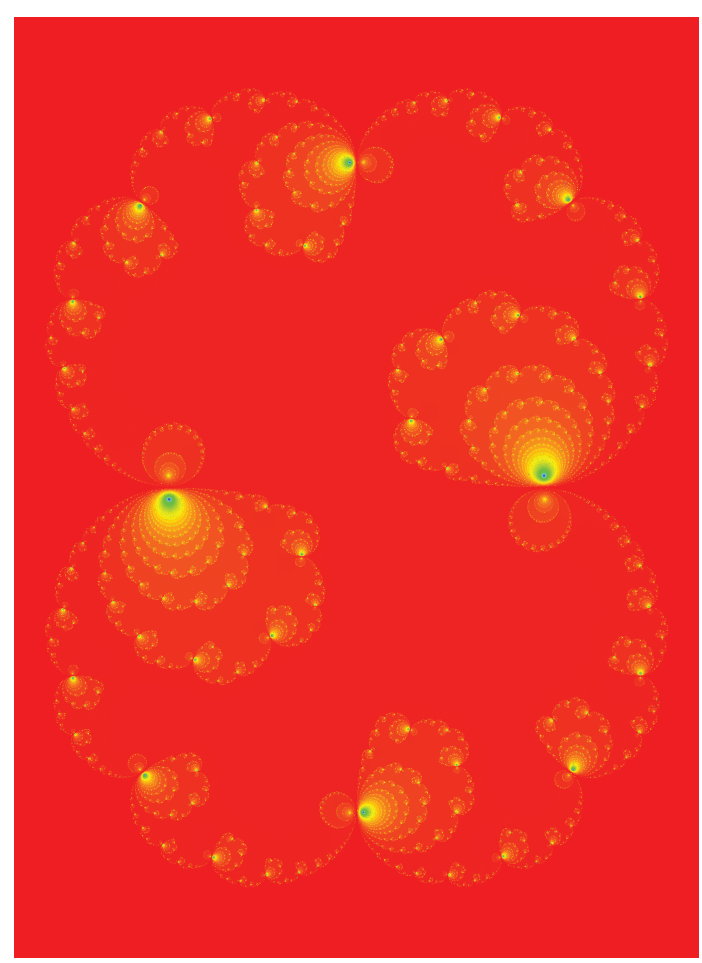

Figure 5. $c \sim 0.25, N=100$.

The picture is generated by iterating $f_{\epsilon} 10,000$ times. Note that this corresponds to iterating $\mathcal{L}_{\alpha, f}$ only 20 times, a rather small number, which explains the occurrence of the faulty black spots. If for the sake of comparison we use the same function $f$ and phase $\alpha$, but use $N=100$, then we get

$$
f_{\epsilon}(z)=z^{2}+0.250986+0.0000394469 i .
$$

Generating the computer picture for the same 10,000 iterates, which now corresponds to the considerably larger 100 iterates of $\mathcal{L}_{\alpha, f}$, gives Figure 5 . We note that the illustration is almost identical to Figure 3, except that the black spots are no longer visible. Even though $N=100$ gives a less accurate approximation of $\mathcal{L}_{\alpha}$, the computer picture generated by iterating 10,000 times provides a better illustration for the dynamical behavior of $\mathcal{L}_{\alpha}$.

Let us end this discussion by indicating why the Lavaurs Theorem implies parabolic implosion. Choose $z \in \mathcal{B}_{f}$. We wish to show that there exist arbitrarily small perturbations $f_{\epsilon}$ of $f$ for which there are points in the Julia set $J\left(f_{\epsilon}\right)$ arbitrarily close to $z$.

Write $\zeta_{0}=\varphi_{f}(z)$, and let $\zeta_{1} \in \mathbb{C}$ be such that $\psi_{f}\left(\zeta_{1}\right)=$ $w \in \partial \mathcal{B}_{f}$. We let $\alpha=\zeta_{1}-\zeta_{0}$, and for $N \in \mathbb{N}$ we define $\epsilon_{N}=\pi /(N-\alpha)$. We recall that with these choices of $\epsilon_{N}$ the maps $f_{\epsilon_{N}}^{\circ N}$ converge to $\mathcal{L}_{\alpha, f}$ uniformly on compact subsets of $\mathcal{B}_{f}$. Further observe that

$$
\mathcal{L}_{\alpha, f}(z)=\psi_{f} \circ \tau_{\alpha} \circ \varphi_{f}(z)=w \in \partial \mathcal{B}_{f} \subset J_{f} .
$$

Now let $U$ be an arbitrarily small open neighborhood of $z$. It follows that $\mathcal{L}_{\alpha, f}(U)$ gives a neighborhood of $w$; hence 
by uniform convergence $f_{\epsilon_{N}}^{N}(U)$ still contains a fixed neighborhood $V$ of $w$ for $N$ sufficiently large. Now recall that the dependence of the Julia set on the parameter $\epsilon$ is lower semicontinuous. Hence for $\epsilon_{N}$ small (which corresponds to $N$ large), the Julia set $J\left(f_{\epsilon_{N}}\right)$ must intersect $V$. Invariance of the Julia set implies that $J\left(f_{\epsilon_{N}}\right)$ must intersect $U$ for $N$ sufficiently large.

Since the above holds for any $z \in \mathcal{B}_{f}$ we have indicated a proof, using the Lavaurs Theorem and properties of the Fatou coordinates as givens, of the following general result:

Theorem 2 (Parabolic implosion). Let $f=f_{0}$ be a rational function with a parabolic fixed point at the origin, written locally as $f(z)=z+z^{2}+O\left(z^{3}\right)$. Denote the parabolic basin by $\mathcal{B}_{f}$ and by $\overline{\mathcal{B}_{f}}$ its closure. Let $\left\{f_{\lambda}\right\}$ be an analytically varying oneparameter family of rational functions for which the parabolic fixed point is not persistent. Then

$$
\overline{\mathcal{B}_{f}} \subset \bigcap_{\delta>0} \overline{\bigcup_{|\lambda|<\delta} J\left(f_{\lambda}\right)}
$$

Thus in general the Julia set varies lower semicontinuously but not continuously at $f_{0}$.

Note that for the quadratic polynomial $f=p_{1 / 4}$ we actually obtain an equality here, due to the fact that the complement of $\mathcal{B}_{p_{1 / 4}}$ equals the basin of infinity. In general this will not be the case.

\section{Extensions and Applications of the Lavaurs Theorem}

In this section we turn our focus to the family of quadratic polynomials. By conjugating with affine transformations the family of quadratic maps can be parametrized by $p_{c}(z)=z^{2}+c$ with complex parameter $c$. A crucial role in the dynamics of rational functions is played by the orbits of critical points, i.e., points where the derivative vanishes. These are exactly the points where the function is not locally invertible. For the polynomial $p_{c}$ the orbit of the unique critical point $z=0$ is therefore of singular importance. If this critical orbit is bounded, the Julia set is connected, while if the critical orbit is unbounded, the Julia set is completely disconnected and therefore a Cantor set. This dichotomy motivates the definition of the set

$$
\mathcal{M}:=\left\{c:\left\{p_{c}^{n}(0)\right\}_{n \in \mathbb{N}} \text { bounded }\right\},
$$

known as the Mandelbrot set. Note that the characterization in terms of the critical orbit is useful for computer illustrations, such as Figure 6.

Let us make a few more comments regarding the Mandelbrot set, without being too precise. First, whereas the Mandelbrot set is defined in terms of quadratic polynomials, it turns out to be a universal object; that is, it arises whenever one studies parameter spaces of rational maps. It is thus a central object in complex dynamical systems,

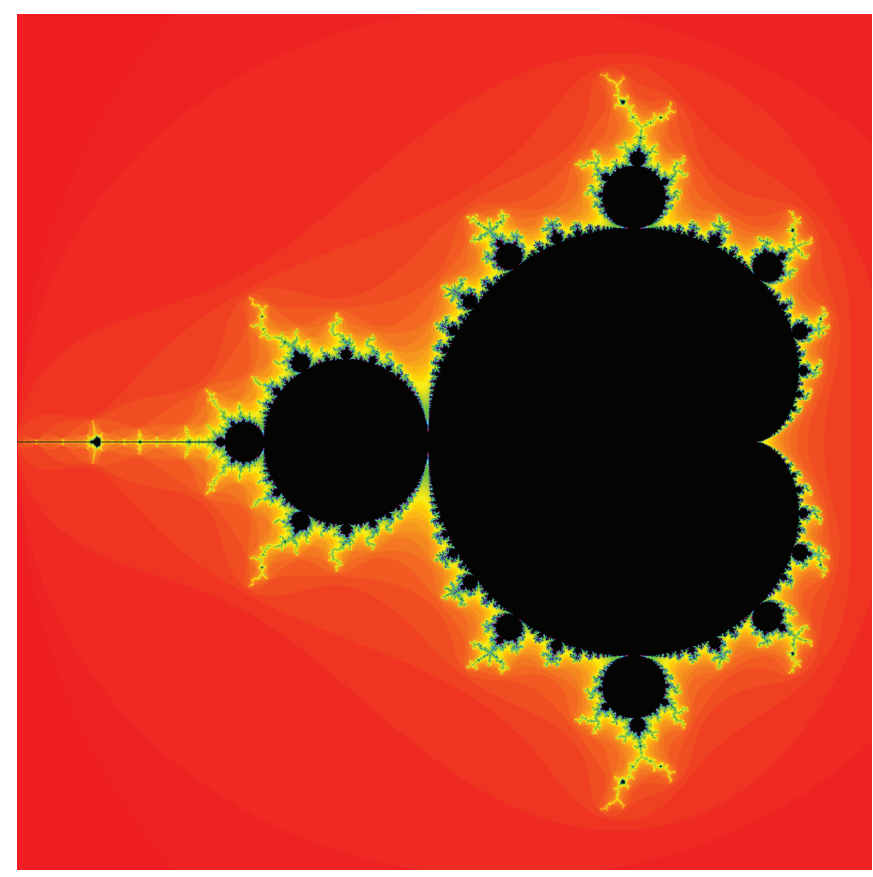

Figure 6. Mandelbrot set.

and has been studied in great detail. One of the first results regarding the Mandelbrot set is that it is connected (see Douady and Hubbard [9]). Still open, and considered one of the main open problems in the field, is whether the Mandelbrot set is locally connected.

The boundary of the Mandelbrot set is exactly equal to the set where the dynamics of $p_{c}$ is not structurally stable. It is referred to as the bifurcation locus. It turns out that there is a strong relationship between the boundary of the Mandelbrot set near a parameter $c$, and the Julia set of the parameter $J_{p_{c}}$, an observation that will be important for the discussion below. Compare for example the boundary near $c=0.25$ illustrated in Figure 7 to the Julia set depicted in Figure 2. This relationship is one of the many intricate reasons why understanding the dynamics of quadratic polynomials is so closely related to understanding the boundary of the Mandelbrot set, and vice versa.

A beautiful result obtained by Shishikura [18] (see also McMullen for another proof [16]) gives the existence of parameters $c$ for which the Julia set $J_{p_{c}}$ has Hausdorff dimension equal to 2 . Note that 2 is the largest possible dimension for any subset of $\mathbb{C}$, trivially assumed by sets with interior. The fact that such naturally occurring sets $J_{p_{c}}$ without interior can have dimension 2 may come as a surprise. Moreover, a consequence of the relationship hinted at above is that the boundary of the Mandelbrot also has Hausdorff dimension 2.

More recently Buff and Cheritat [7] proved that Julia sets of quadratic polynomials can have positive Lebesgue measure, which is considerably stronger than having Hausdorff dimension 2. The relationship between the 


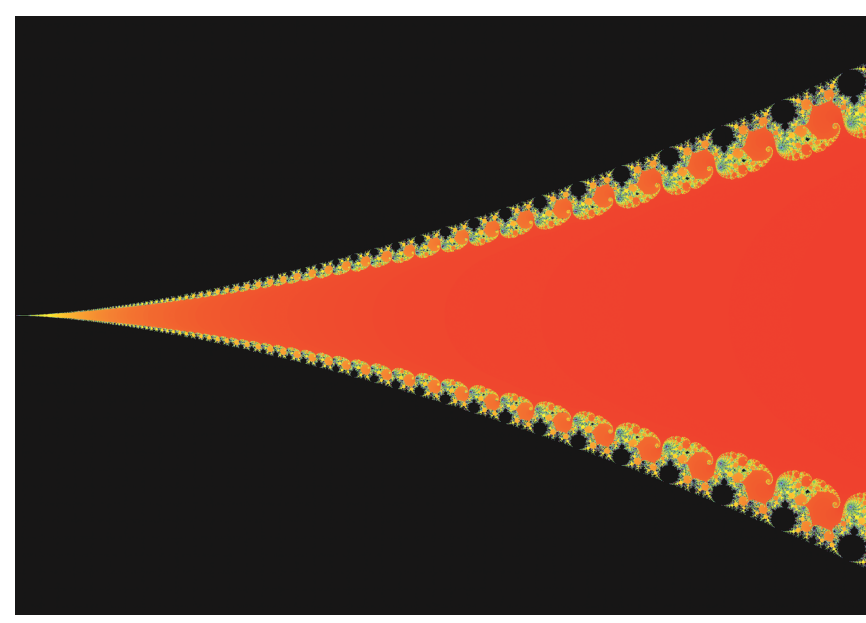

Figure 7. Zooming in on $c=0.25$.

Mandelbrot set and Julia sets is however not strong enough to deduce the same result for the boundary of the Mandelbrot set. See the results of Avila and Lyubich [3] for further discussion regarding Hausdorff dimensions and measures occurring for quadratic polynomials.

The strategy of Shishikura's proof is to construct quadratic polynomials with Hausdorff dimension arbitrarily close to 2 . These polynomials will have a fixed point with multiplier $\alpha$ close to some $\exp (2 \pi i p / q)$ with suitable integers $p$ and $q$. Shishikura then constructs a hyperbolic subset (with Hausdorff dimension close to 2) of the Julia set. The main tool for constructing the hyperbolic set and estimating its dimension is the theory of parabolic bifurcations. The result follows after taking an appropriate limit.

\section{Parabolic Implosion in Higher Dimensions}

In recent decades there has been significant interest in the dynamics of polynomial and rational maps in several complex variables. Compared to the deep existing theory of one-dimensional systems, our current understanding of iteration problems in several variables remains poor. We wish to point out that this is not entirely our fault: it has become clear that intricate new phenomena arise in higher dimensions, phenomena that make a rigorous analysis of the systems much more complicated.

An example of the difficulties that arise in higher dimensions already occurs when trying to find the right analogue of the Lavaurs Theorem. For example, the correct interpretation of parabolic cycles, central in our earlier discussion, is not at all clear in higher dimensions. In dimension $n$, the Jacobian derivative at a fixed point will typically have $n$ distinct eigenvectors, with corresponding eigenvalues. Any number of those eigenvalues could be roots of unity. What makes the situation even more complicated is that resonance phenomena are guaranteed to occur when one eigenvalue is a root of unity, and they can also occur even when no eigenvalue is a root of unity.
For the simplest generalization of a parabolic fixed point, exactly one eigenvalue is assumed to be 1, while all other eigenvalues have norm strictly greater than 1 (or equivalently, strictly smaller than 1 , since these systems are locally invertible). We call such fixed points semiparabolic. Some of the bifurcation phenomena that can occur for semiparabolic fixed points have recently been described by Bedford, Smillie, and Ueda [5], who proved a twodimensional generalization of the Lavaurs Theorem. The analogues of parabolic petals and Fatou-Leau coordinates for semiparabolic of order-1 fixed points were constructed earlier by Ueda [20].

A general goal in dynamical systems, closely related to the stability problem discussed earlier, is to describe the dynamical behavior of "typical" systems. An early attempt was formulated in Smale's Program. Smale suggested that hyperbolic systems, which should be seen as the simplest possible systems, are open and dense. Although Smale's Program did not survive long in the real setting (it was proven to be false in the early 1970s, amongst others by Smale), the density of hyperbolicity remains open for families of one-dimensional polynomials, and is generally thought to hold true for the quadratic polynomials.

In higher dimensions density of hyperbolicity is known to be false; a first construction is due to Buzzard [8]. Buzzard proved that there exist open sets in the complex parameter space of polynomial automorphisms, all of which have homoclinic tangencies, i.e., tangencies between stable and unstable manifolds of a saddle fixed point. Such tangencies prevent hyperbolicity; hence persistent tangencies show that hyperbolicity is not dense.

Motivated by these results one might wonder if there is a larger set of dense automorphisms that can still be understood relatively well. Palis suggested that the parameters with homoclinic tangencies might be dense inside the maps that are persistently nonhyperbolic. Although the Palis Program also turned out to be false in greater generality, it remains unsettled for the family of polynomial automorphisms of $\mathbb{C}^{2}$.

In [10] Dujardin and Lyubich proved a density property for parameters with nonpersistent homoclinic tangencies in the bifurcation locus for families of polynomial automorphisms of $\mathbb{C}^{2}$ with nontrivial dynamics. Their result relies in an essential way on implosion techniques for semiparabolic fixed points of arbitrary order of contact.

At the other extreme, one can consider as generalizations of parabolic fixed points the case where all generalized eigenvalues are equal to 1 . The most studied case is that of maps tangent to the identity. The existence of petals and Fatou-Leau coordinates for such maps has been studied in classical papers [1,11], a description that has recently been completed in [13]. The (dis)continuity of the dynamics near a tangent to the identity parameter has recently 
been considered by Bianchi [6], who proved a version of the Lavaurs Theorem. Using this generalization, Bianchi proves the discontinuity of the filled Julia set for perturbations of so-called regular polynomials.

Yet another example of a phenomenon that occurs only in higher dimensions is that of wandering Fatou components. A Fatou component is a connected component of the Fatou set. A Fatou component is mapped exactly onto a Fatou component, and the layout of these components together with the action of the map paints an insightful combinatorial picture of the dynamics. For rational functions in one complex variable, Sullivan [19] showed that each Fatou component is preperiodic. The possible periodic Fatou components have been precisely described in the works of Fatou, Siegel, and Herman: The action of the map on an invariant component either attracts all orbits to a given fixed point (attracting if it lies in the interior, parabolic if it lies on the boundary) or induces a circle action.

In higher dimensions there has been significant progress in describing invariant Fatou components [4, 14], but it was recently shown that there can exist wandering domains [2]. Astorg, Buff, Dujardin, Peters, and Raissy have used parabolic implosion to prove the existence of a wandering Fatou component in two dimensions [2]. Based on an idea of Lyubich, they use parabolic implosion techniques on families of polynomial skew products to construct wandering domains. More precisely, the idea is to combine slow convergence to an invariant parabolic fiber and parabolic transition in the fiber direction, to produce orbits shadowing those of a Lavaurs map.

The new phenomena arising in higher dimensions create important challenges for the years to come. Obtaining a more thorough understanding of the behavior near parabolic parameters will form an important step in tackling these challenges.

\section{References}

[1] Marco Abate, Fatou flowers and parabolic curves, Complex analysis and geometry, Springer Proc. Math. Stat., vol. 144, Springer, Tokyo, 2015, pp. 1-39, DOI 10.1007/978-4-43155744-9_1 MR3446744

[2] Matthieu Astorg, Xavier Buff, Romain Dujardin, Han Peters, and Jasmin Raissy, A two-dimensional polynomial mapping with a wandering Fatou component, Ann. of Math. (2) 184 (2016), no. 1, 263-313, DOI 10.4007/annals.2016.184.1.2. MR3505180

[3] Artur Avila and Mikhail Lyubich, Hausdorff dimension and conformal measures of Feigenbaum Julia sets, J. Amer. Math. Soc. 21 (2008), no. 2, 305-363, DOI 10.1090/S0894-034707-00583-8 MR2373353

[4] Eric Bedford and John Smillie, Polynomial diffeomorphisms of $\mathbf{C}^{2}$. II. Stable manifolds and recurrence, I. Amer. Math. Soc. 4 (1991), no. 4, 657-679, DOI 10.2307/2939284 MR1115786
[5] Eric Bedford, John Smillie, and Tetsuo Ueda, Semiparabolic bifurcations in complex dimension two. Comm. Math. Phys. 350 (2017), no. 1, 1-29, DOI 10.1007/s00220017-2832-y MR3606468

[6] Fabrizio Bianchi, Parabolic implosion for endomorphisms of $\mathbb{C}^{2}$, J. Eur. Math. Soc. (JEMS) 21 (2019), no. 12, 3709-3737, DOI 10.4171/JEMS/911. MR4022713

[7] Xavier Buff and Arnaud Chéritat, Ensembles de Julia quadratiques de mesure de Lebesgue strictement positive (French, with English and French summaries), C. R. Math. Acad. Sci. Paris 341 (2005), no. 11, 669-674, DOI 10.1016/j.crma.2005.10.001 MR2183346

[8] Gregery T. Buzzard, Infinitely many periodic attractors for holomorphic maps of 2 variables, Ann. of Math. (2) 145 (1997), no. 2, 389-417, DOI 10.2307/2951819 MR1441881

[9] Adrien Douady and John Hamal Hubbard, Itération des polynômes quadratiques complexes (French, with English summary), C. R. Acad. Sci. Paris Sér. I Math. 294 (1982), no. 3, 123-126. MR651802

[10] Romain Dujardin and Mikhail Lyubich, Stability and bifurcations for dissipative polynomial automorphisms of $\mathbb{C}^{2}$, Invent. Math. 200 (2015), no. 2, 439-511, DOI 10.1007/s00222-014-0535-y MR3338008

[11] Monique Hakim, Analytic transformations of $\left(\mathbf{C}^{p}, 0\right)$ tangent to the identity, Duke Math. J. 92 (1998), no. 2, 403428, DOI 10.1215/S0012-7094-98-09212-2 MR1612730

[12] P. Lavaurs, Systemes dynamiques holomorphes: explosion de points périodiques paraboliques, $\mathrm{PhD}$ thesis, Paris 11, 1989.

[13] L. López-Hernanz, J. Raissy, J. Ribón, and F. Sanz-Sánchez, Stable manifolds of two-dimensional biholomorphisms asymptotic to formal curves, preprint, arXiv 1710.037282017.

[14] Mikhail Lyubich and Han Peters, Classification of invariant Fatou components for dissipative Hénon maps, Geom. Funct. Anal. 24 (2014), no. 3, 887-915, DOI 10.1007/s00039-014-0280-9 MR3213832

[15] R. Mañé, P. Sad, and D. Sullivan, On the dynamics of rational maps, Ann. Sci. École Norm. Sup. (4) 16 (1983), no. 2, 193-217. MR732343

[16] Curtis T. McMullen, Hausdorff dimension and conformal dynamics. II. Geometrically finite rational maps, Comment. Math. Helv. 75 (2000), no. 4, 535-593, DOI 10.1007/s000140050140. MR1789177

[17] Mitsuhiro Shishikura, Bifurcation of parabolic fixed points, The Mandelbrot set, theme and variations, London Math. Soc. Lecture Note Ser., vol. 274, Cambridge Univ. Press, Cambridge, 2000, pp. 325-363. MR1765097

[18] Mitsuhiro Shishikura, The Hausdorff dimension of the boundary of the Mandelbrot set and Julia sets, Ann. of Math. (2) 147 (1998), no. 2, 225-267, DOI 10.2307/121009 MR1626737

[19] Dennis Sullivan, Quasiconformal homeomorphisms and $d y$ namics. I. Solution of the Fatou-Julia problem on wandering domains, Ann. of Math. (2) 122 (1985), no. 3, 401-418, DOI 10.2307/1971308 MR819553 
[20] Tetsuo Ueda, Local structure of analytic transformations of two complex variables. II, J. Math. Kyoto Univ. 31 (1991), no. 3, 695-711, DOI 10.1215/kjm/1250519724. I: J. Math. Kyoto Univ. 26 (1986), 233-261, MR849219 MR1127094

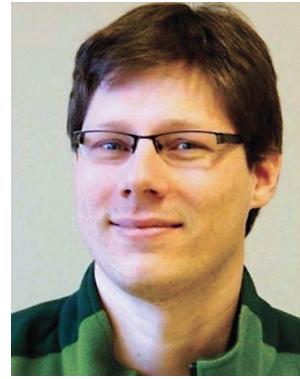

Han Peters

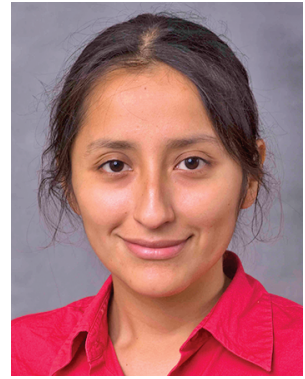

Liz Vivas

\section{Credits}

All figures in this article were created by the authors using the "JuliaSetPlot" command in Mathematica.

Photo of Han Peters is courtesy of Tiny Dekker.

Photo of Liz Vivas is courtesy of Purdue University.

\section{FEATURED TITLES FROM 品 HINDUSTAN BOOK AGENCY}

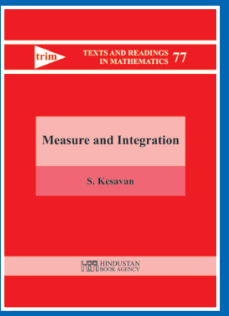

Measure and Integration

S. Kesavan

This book deals with topics usually studied in a master's or graduate level course on the theory of measure and integration. It starts with the Riemann integral and points out some of its shortcomings which motivate the theory of measure and the Lebesgue integral

2019; 252 pages; Hardcover; ISBN: 978-93-86279-77-4 List US\$45; AMS members US\$36; Order code HIN/77

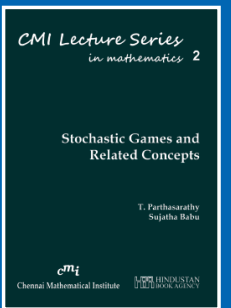

Stochastic Games and Related Concepts T. Parthasarathy and Sujatha Babu This set of lecture notes is based on a series of ten lectures given by T. Parthasarathy at the Chennai Mathematical Institute. Topics in matrix and bimatrix games, stochastic games (finite, infinite, and undiscounted) and cooperative games are covered.

2020; 156 pages; Softcover; ISBN: 978-93-86279-79-8;

List US\$56; AMS members US\$44.80; Order code HIN/78

Titles published by the Hindustan Book Agency (New Delhi, India) include studies in advanced

mathematics, monographs, lecture notes, and/or conference proceedings on current topics of interest.

Discover more books at bookstore.ams.org/hin

Publications of Hindustan Book Agency are distributed within the Americas by the American Mathematical Society. Maximum discount of $20 \%$ for commercial channels.

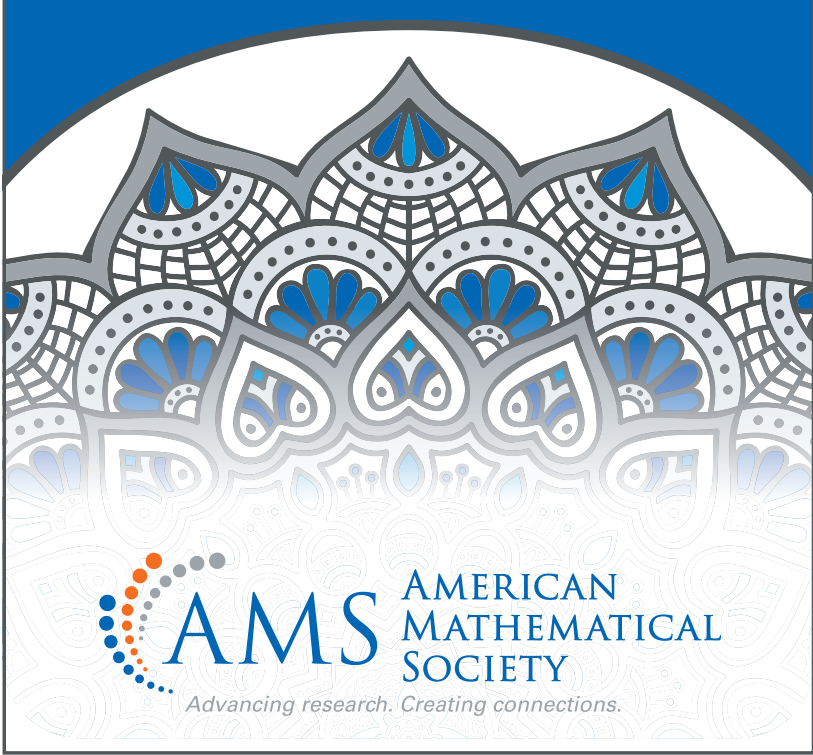

